\title{
Paranasal Carcinoma: A Case Series
}

\author{
Shakil Aqil ${ }^{1}$, Iqra Zakir ${ }^{*}$, Hamdan Pasha ${ }^{1}$ and Zubair Khan ${ }^{1}$ \\ ${ }^{1}$ Department of E.N.T-Head and Neck Surgery, Liaquat National Hospital and Medical College, Karachi, Pakistan
}

\begin{abstract}
Objective: To emphasize the importance of early diagnosis of cancer of paranasal sinus.

Method: This is retrospective case series done in Liaquat National Hospital. Data were retrieved from August to October 2020.

Results: Data of a total of 5 patients with sinonasal cancer was reviewed. Four patients presented with extensive disease. Three patients were male and two were female. The age of patients was between 40 and 70 years. All these five patients underwent surgery. Four patients were referred for concurrent chemoradiotherapy (CCRT) and one patient expired after surgery.

Conclusion: Malignant tumors of the nose and sinuses are rare constituting $3 \%$ of head and neck malignancies. The early symptoms are relatively innocuous and are often ignored by the patient, their general practitioners and even ENT surgeons leading to a delayed diagnosis and treatment. Although paranasal tumors are rare they should be in our differential.
\end{abstract}

Keywords: Paranasl carcinoma, sinonasal cancer, malignant tumors, nose, diagnose.

\section{INTRODUCTION}

Sinonasal tumors are rare, but due to bad prognosis and morbidity, these tumors deserve great attention. Earlier diagnosis, better local control, and the development of new and better therapeutic modalities can improve the management and prognosis but unfortunately, late diagnosis of the malignant tumors in this anatomic area confound these goals.

Diagnosis is mostly delayed because early symptoms are misleading or ignored. Symptoms usually mimic benign diseases. Local control is difficult due to the extension of the tumor to vital structures at the cribriform plate, pterygomaxillary space, orbital apex, sphenoid, and nasopharynx. The paucity of lymphatic drainage from the sinuses results in a low incidence (approximately $10 \%$ ) of cervical lymphadenopathy.

The major challenge to the head and neck surgeon is to improve the chances of cure and yet provide functional and cosmetic reconstruction. This case series will help otolaryngologists to update their knowledge about this rare disease so that early diagnosis and treatment can be done.

\section{MATERIALS AND METHODS}

We reviewed the record of five patients who presented in Liaquat national hospital and were diagnosed with paranasal tumors from august 2020 till October 2020. Medical, radiological and pathological records were reviewed in detail.

*Corresponding Author: Iqra Zakir, Department of E.N.T-Head and Neck Surgery, Liaquat National Hospital and Medical College, Karachi, Pakistan; Email: iqrazakir00@gmail.com

Received: October 06, 2020; Revised: January 18, 2021; Accepted: February 17, 2021 DOI: https://doi.org/10.37184/Intbj.2708-7808.2.13
Details of all these five cases are following.

\section{Case 1:}

A 40- year old woman with no known comorbid came to opd with a complaint of swelling on the right side of the face for 2 months. The swelling was progressively increasing in size and associated with nasal obstruction. There was no history of addiction or any exposure to carcinogens. On examination approx. $5 \times 4 \mathrm{~cm}$ firm swelling was present in the right maxillary region starting superiorly from the infra-orbital margin, inferiorly up to nasolabial angle, medially involving right nasal cavity and laterally up to the zygomatic arch, overlying skin was erythematous but no tethering present. The swelling was present on the right eye and the patient was not able to open it, although the vision of the right eye was normal (Fig. 1).

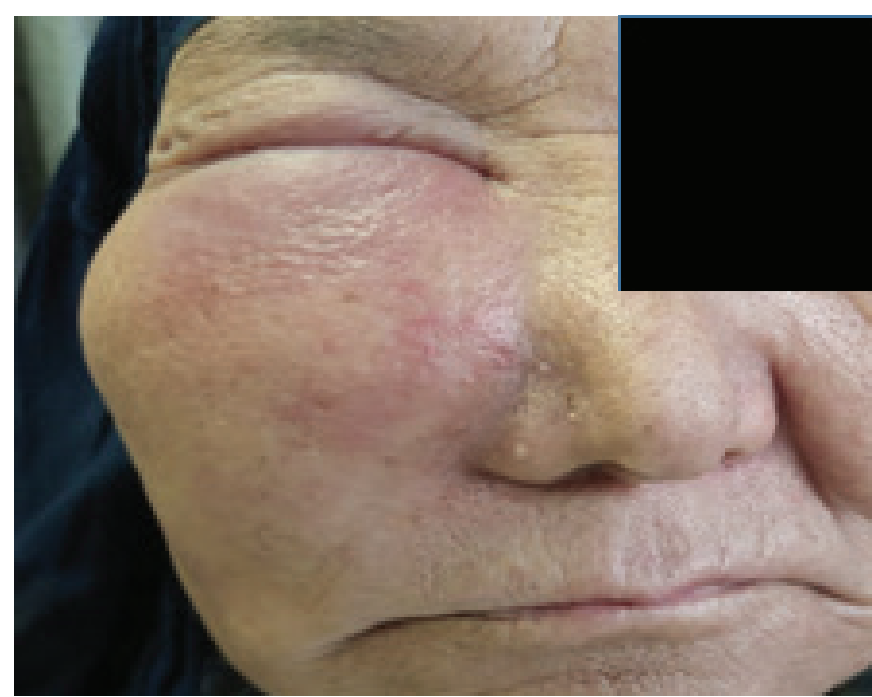

Fig. (1): Large swelling involving right maxillary region and right eye. 
CT scan paranasal sinuses showed, ill-defined heterogeneously enhancing infiltrating lesion approx. 67 $\mathrm{mm} \times 51 \mathrm{~mm}$ x $67 \mathrm{~mm}$ involving maxillary sinus, anterior and posterior ethmoid and right nasal cavity. The lesion is extending to the adjacent anteromedial aspect of the right orbit cavity, medially lesion reached nasal septum, anteromedially lesion extending to the subcutaneous soft tissue of right cheek. Significant destructive changes of underlying maxillary and ethmoidal bones were noted. No intracranial extension of the lesion is noted (Figs. 2A\&2B). A nasal biopsy was done which showed poorly differentiated large cell neuroendocrine carcinoma. CT chest was done to rule out metastasis but it was unremarkable.
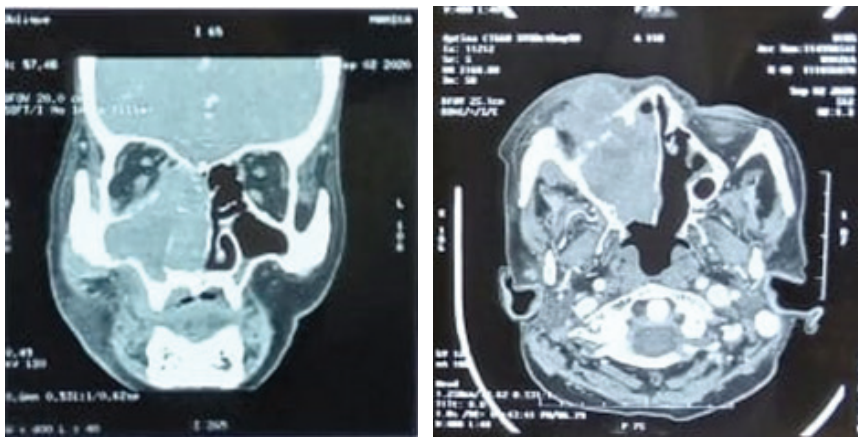

Figs. (2A\&2B): Large mass involving right maxillary sinus, anterior and posterior ethmoid, nasal cavity and orbit with bony destruction of the maxillary sinus and ethmoid sinus.

So right maxillectomy + right orbital exenteration + reconstruction with forehead flap was done. The specimen was shown in (Fig. 3). The postoperative period went uneventful and the patient got discharged on $3^{\text {rd }}$ POD on antibiotics and analgesics. The patient was called for follow-up after 1 week. Final histopathology showed poorly differentiated large cell neuroendocrine carcinoma T4NxMx. All margins were cleared.

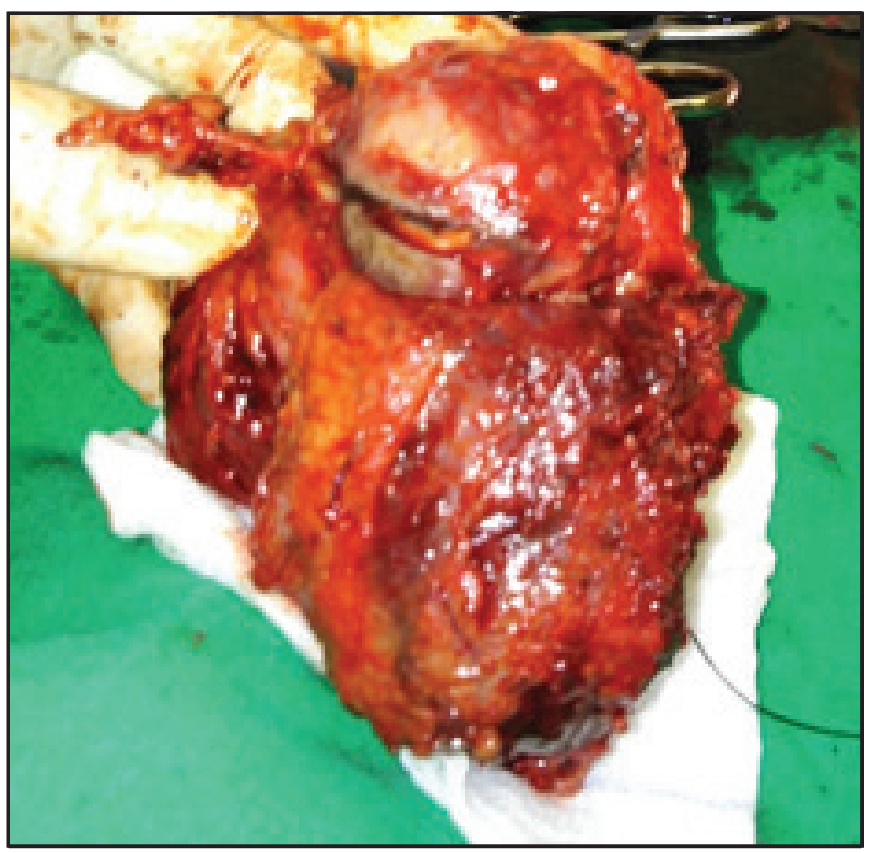

Fig. (3): Specimen of a patient after excision.
Proliferation index Ki67 was approximately 95\%. The case was discussed in the tumor board meeting and concurrent chemoradiation was advised.

\section{Case 2:}

A 70 year- old man with no known comorbid presented in ENT clinic with a complaint of right facial swelling and loss of vision in the right eye for the last 5 months. There was no history of addiction and exposure to chemicals. On examination, the patient had a large firm swelling extending from the medial canthus to the zygoma, adherent to the overlying skin, causing the displacement of the eyeball laterally. There was another swelling below it that spared the overlying skin. There was no vision or light perception in the right eye (Fig. 4).

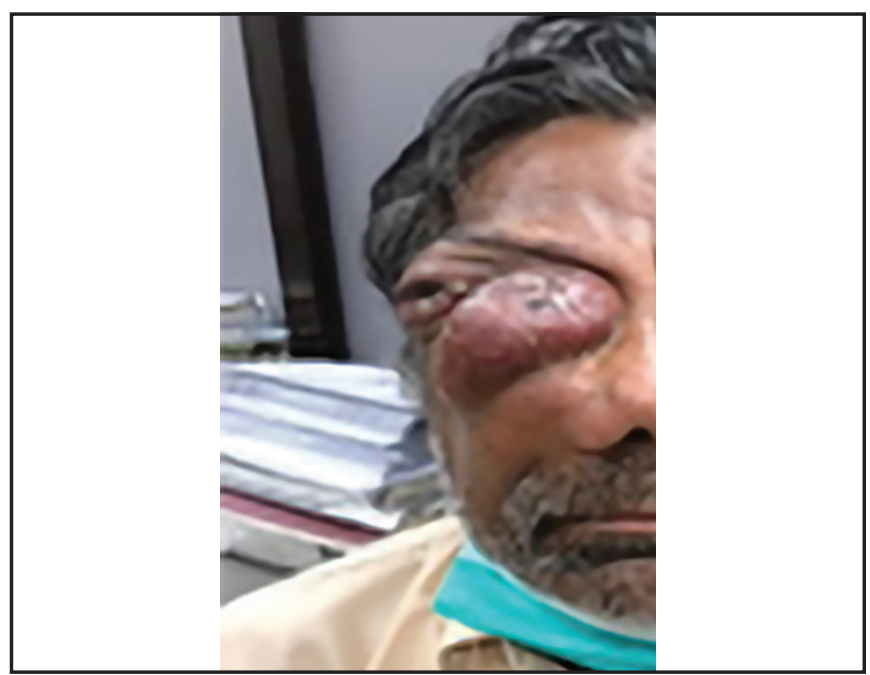

Fig. (4): Large mass involving right medial canthal region and displacing the eye laterally.

The CT scan was done which showed large bulky heterogeneously enhancing mass with internal necrosis involving the right nasal cavity and extending into right maxillary and ethmoid sinuses. The mass was causing erosion and destruction of anterior and medial walls of the right maxillary sinus and lamina papyracea with significant extension into the right orbit, involving medial and inferior recti muscles. Medially it was abutting the nasal septum (Figs. 5A\&5B).
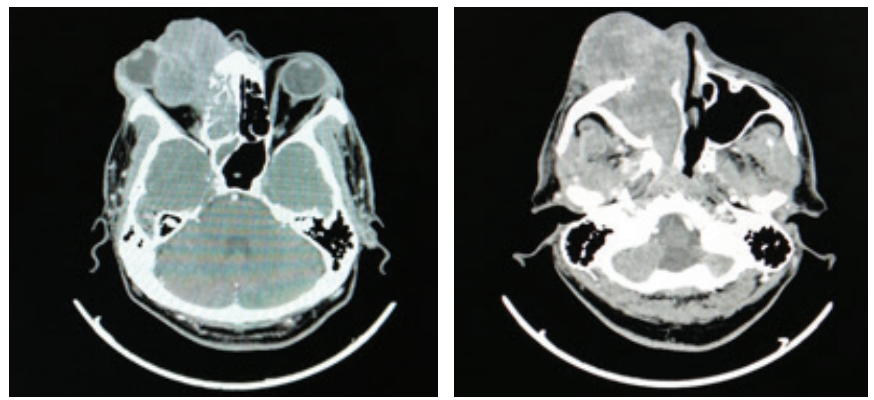

Figs. (5A\&5B): Large mass with internal necrosis involving nasal cavity, maxillary sinus, ethmoid sinus and orbit with bony destruction of maxilla and lamina papyracea.

The biopsy was done which showed well to moderately differentiated squamous cell carcinoma. Surgery was 
planned and he underwent right radical maxillectomy + right orbital Exenteration + clearance of right ethmoidal, frontal and sphenoid sinuses and reconstruction with a free flap. After surgery unfortunately his free flap did not survive and he developed sepsis due to which he expired on $7^{\text {th }}$ POD. Final histopathology showed squamous cell carcinoma T4NxMx. All margins were cleared.

\section{Case 3:}

A 60- year old woman, a known case of hypertension, diabetes mellitus and ischemic heart disease, presented in ENT clinic with a complaint of right nasal blockage for 2 years associated with anosmia, nasal discharge and postnasal dripping. There was no history of addiction and exposure to carcinogens. On examination polypoidal tissue filling the right nasal cavity. Right-sided proptosis was present but vision and eye movements were normal. CT scan of paranasal sinuses was done which showed a polypoidal mass in the right nasal cavity-causing erosion of the septum and protruding into the left nasal cavity. Mass extending into bilateral maxillary, ethmoidal and sphenoidal sinuses causing thinning and re-modeling of bony margins with the widening of bilateral osteomeatal complexes. Posteriorly mass extending into nasopharynx on the right side. It measures approximately $9.0 \times 4.5 \mathrm{~cm}$ in axial dimensions causing thinning and focal erosion of the right lamina papyracea and showing extension into the medial part of the right orbit, compressing and displacing the right medial rectus muscle laterally and causing proptosis. Findings are likely due to sinonasal polyposis (Fig. 6).
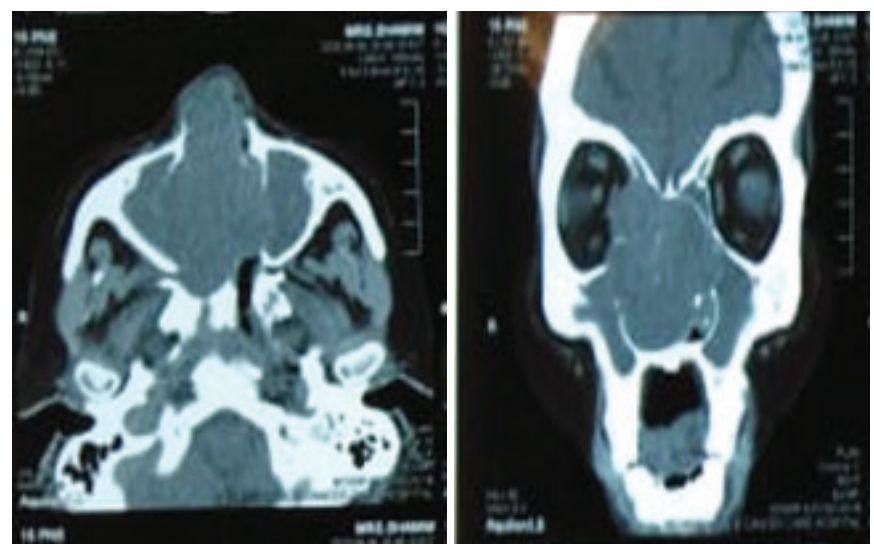

Fig. (6): Large mass involving right nasal cavity, maxillary sinus, ethmoid sinus, sphenoid sinus, nasopharynx and orbit, causing erosion of lamina papyracea.

So, after all baseline investigations of the patient, we planned functional endoscopic sinus surgery. On the table appearance of tissues looked suspicious so sent for the frozen section which reported as invasive malignancy. We changed our plan and did endoscopic removal of a tumor from the bilateral nasal cavity, bilateral maxillary sinuses, bilateral ethmoidal sinuses and right orbit. The patient remained stable in the postop period and was discharged on $2^{\text {nd }}$ POD on antibiotics and analgesics. Final histopathology reported as nonkeratinizing squamous cell carcinoma T4NxMx. All margins were tumor-free. The case was discussed in the tumor board and the patient was referred for concurrent chemoradiation.

\section{Case 4:}

A64-year old man with no known comorbid came with the complaint of left nasal blockage for 3 months associated with nasal discharge and facial pain for 1 month. He was a painter by profession and also had a history of smoking for the last 30 years. On examination ulcerative fungating lesion was found on the left side of the nose in the middle meatus pushing the middle turbinate medially and extending till the floor of the nose. Thick mucus was present on the left side of the nose (Fig. 7).
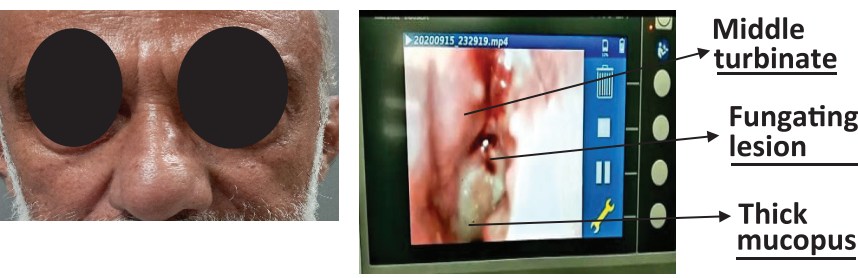

Fig. (7A): Picture of the patient, no external swelling or mass present. (7B): Endoscopic picture of the nose showing fungating lesion.

CT scan showed an extensive heterogeneously enhancing lesion in the left side of the sinonasal passage, its epicenter was in the maxillary sinus, bulging slightly in infratemporal fossa abutting pterygoid muscle, superiorly the mass was involving left ethmoid sinus with bone destruction. Lamina papyracea was also eroded. No intracranial cavity or orbital extension was seen (Fig. 8). Biopsy showed moderate to poorly differentiated squamous cell carcinoma.

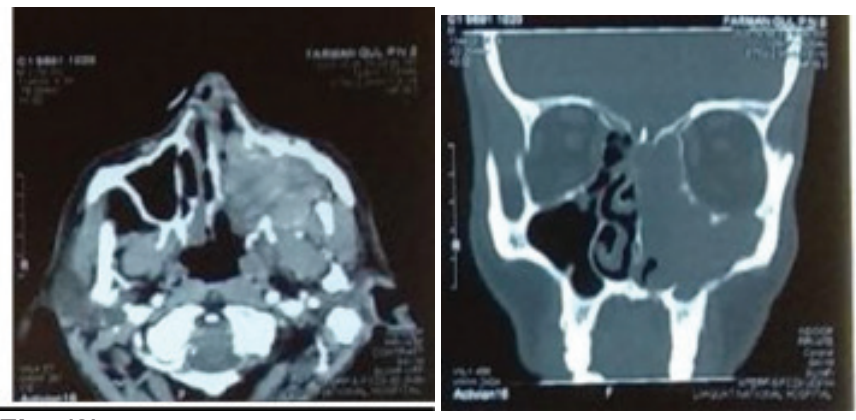

Fig. (8): Large mass involving left maxillary sinus, ethmoid sinus and slightly entering into the infratemporal fossa.

Left total maxillectomy and removal of pterygoid muscle from infratemporal fossa. The specimen is shown in (Fig. 9). The patient remained stable after surgery and was discharged on $3^{\text {rd }}$ POD. The final histopathology

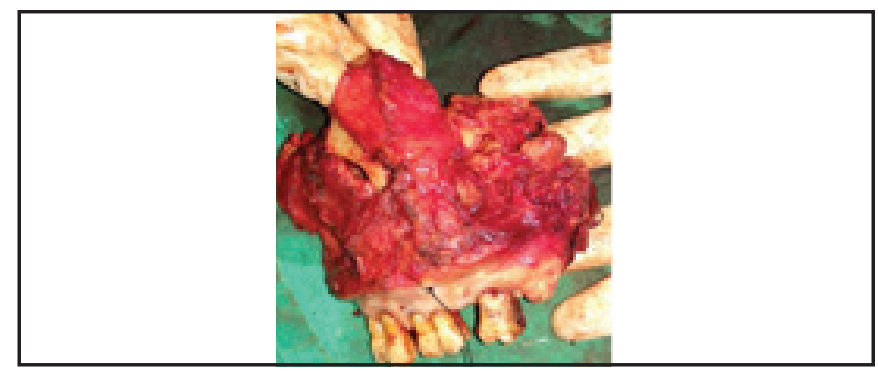

Fig. (9): Specimen after excision. 
showed T4NxMx disease. All margins were cleared. The case was discussed in the tumor board and concurrent chemoradiation was advised.

\section{Case 5:}

A 40-year-old man with no known comorbid presented with a complaint of right nasal obstruction for 3 years. There was no history of smoking or exposure to carcinogens. On examination, a polypoidal mass was filling the right nasal cavity most likely arising from the middle turbinate (Fig. 10).

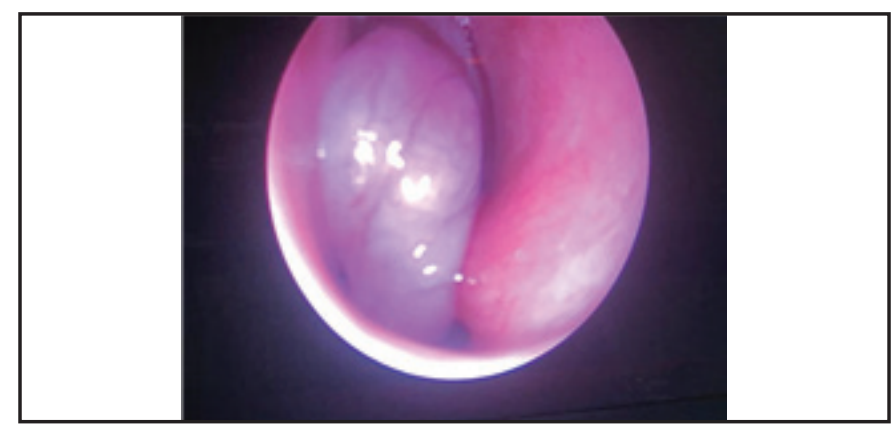

Fig. (10): Endoscopic picture showing a mass in the right nasal cavity.

CT scan of sinuses showed a well-defined polypoidal mass, involving right nasal cavity measures about $3.0 \mathrm{x}$ $1.0 \times 4.0 \mathrm{~cm}$, abutting the nasal septum and middle and inferior turbinate. No extension into the right maxillary sinus, pterygomaxillary fissure and infratemporal fossa (Fig. 11).

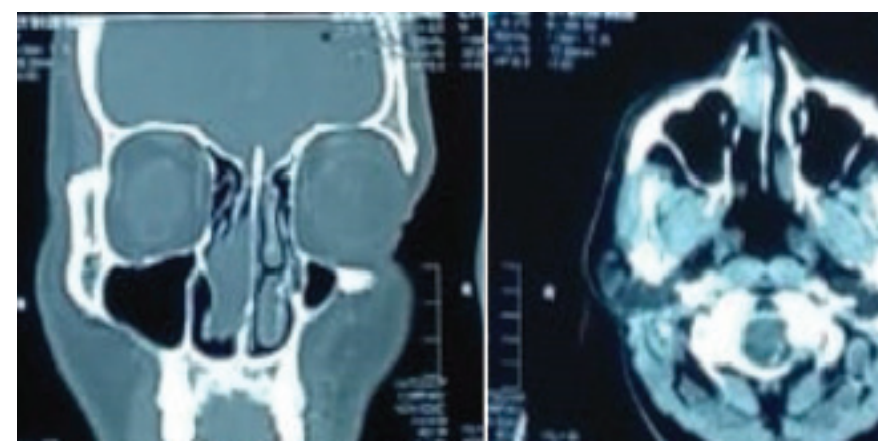

Figs. (11A\&11B): Mass involving right nasal cavity abutting inferior and middle turbinate.

Endoscopic removal of nasal mass was done and the whole mass was excised (Fig. 12).

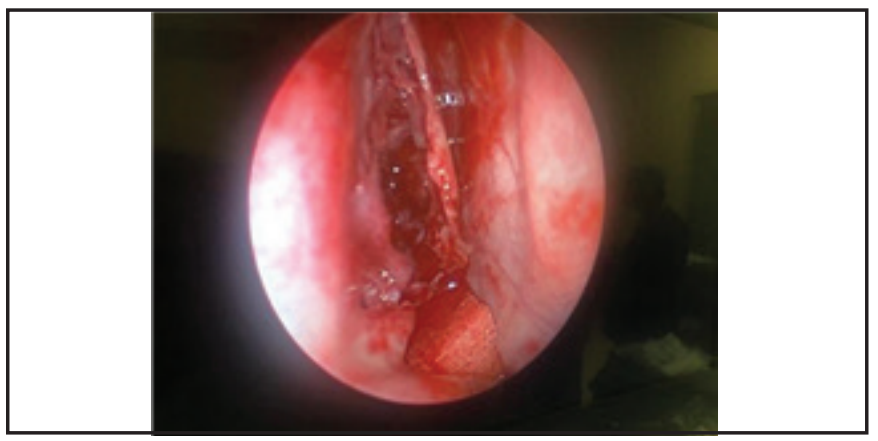

Fig. (12): Picture after removing all tumors.
Histopathology showed high-grade large cell neuroendocrine carcinoma. All margins were cleared. Proliferation index Ki67 was in the order of $25 \%$. The case was discussed in the tumor board and a PET scan was advised after 4 weeks of the surgery to rule out metastasis.

\section{DISCUSSION}

The incidence of nose and paranasal sinus tumors is 0.5 to $1 \%$ per 100,000 per year [1]. They account for $3 \%$ of upper aerodigestive tract tumors [2]. Most of these tumors develop in the fifth and sixth decades of life. Sinonasal tumors are more common in men [3]. The most common histological type is squamous cell carcinoma $60 \%$ followed by adenocarcinoma $9 \%$, adenoid cystic carcinoma $5 \%$, neuroendocrine tumors $5 \%$, olfactory neuroblastoma $<5 \%$, melanoma $3.6 \%$, and then comes very rare tumor's like undifferentiated sinonasal carcinoma, haemangiopericytomas, lymphomas and sarcomas. Maxillary sinus tumors are the most common (60 percent) followed by the nasal cavity (20 percent), ethmoid sinuses (5 percent) and rarely frontal and sphenoid sinuses (1 percent) [4].

Tumors of the nose and paranasal sinuses cause progressive nasal blockage, epistaxis and obstruction of sinus outflow. Distortion of the nose develops when the disease gets advanced. The presentation of most sinus malignancies is considerably delayed as early symptoms mimic benign disease. The sinus from where the tumor originates is filled with tumor and symptoms are caused when the tumor causes erosion of its walls and extends beyond. The most common symptoms are nasal blockage, nasal bleeding, palate mucosal ulceration, loose teeth, facial swelling, facial pain, diplopia, and proptosis. Dulguerov et al. found that patients with T4 stage disease have the lowest survival rate [5]. If the tumor invades critical structures like meninges, brain parenchyma, skull base, orbit, cavernous sinus and infratemporal fossa then the prognosis is not good and the survival rate is significantly reduced [6].

Computed tomography and magnetic resonance imaging are routine imaging studies to assess the extent of a tumor. The prognostic factors that affect locoregional recurrence include an advanced stage of tumors, aggressive histological types and cervical lymphadenopathy. Treatment options depending upon the stage of the tumor, mainly consist of surgery combined with radiotherapy and chemotherapy [7]. Radical resection is recommended for early-stage tumors. An alternative approach used for early staged tumors is endoscopic surgery which achieves excellent results [8]. Multimodality treatment is needed for advanced-stage tumors for better locoregional control.

\section{CONCLUSION}

Sinonasal nasal carcinomas are very rare and most of the patients present very late with advanced disease 
because symptoms are very confusing and mimic benign disease. In our case series four out of five patients presented with very advanced disease. Health education on early consultation and efforts at early detection of the disease is needed to achieve a cure.

\section{ETHICS APPROVAL}

For this type of study formal consent is not required.

\section{CONSENT FOR PUBLICATION}

Not applicable.

\section{FUNDING}

None.

\section{CONFLICT OF INTEREST}

The authors declare no conflict of interest.

\section{ACKNOWLEDGEMENTS}

None

\section{REFERENCES}

1. Dulguerov $P$, Jacobsen MS, Allal AS, Lehmann W, Calcaterra T. nasal and paranasal sinus carcinoma: are we making progress? Cancer 2001; 92: 3012-29.
2. Rice DH, Stanley Jr. RB. Surgical therapy of tumors of the nasal cavity, ethmoid sinus, and maxillary sinus. In: Panje W, Eds. Comprehensive Management of Head and Neck Tumors. $2^{\text {nd }}$ ed. Philadelphia: Saunders 1999; pp. 558-81.

3. Homma A, Hayashi R, Matsuura K, et al. Lymph Node Metastasis in T4 Maxillary Sinus Squamous Cell Carcinoma: Incidence and Treatment Outcome. Ann Surg Oncol 2014; 21: 1706-10.

4. Kazi M, Awan S, Junaid M, Qadeer S, Hassan NH. Management of sinonasal tumors: prognostic factors and outcomes: a 10 year experience at a tertiary care hospital. Indian J Otolaryngol Head Neck Surg 2013; 65: 155-9.

5. Sanghvi S, Khan MN, Patel NR, Yeldandi S, Baredes S, Eloy JA. Epidemiology of sinonasal squamous cell carcinoma: a comprehensive analysis of 4994 patients. Laryngoscope 2014; 124: $76-83$

6. Michel J, Fakhry N, Mancini J, et al. Sinonasal squamous cell carcinomas: clinical outcomes and predictive factors. Int $\mathrm{J}$ Oral Maxillofac Surg 2014; 43: 1-6.

7. Kano S, Hayashi R, Homma A, et al. Effect of local ectension sites on survival in locally advanced maxillary sinus cancer. Head Neck 2014; 36: 1567-72.

8. Saedi B, Aghili M, Motiee M, Valadkhani S, Niazi AB, Safavi A Surgical outcomes of malignant sinonasal tumors: open versus endoscopic surgical approaches. J Laryngol Otol 2014; 128: 78490.

\section{ANSWER TO QUESTION \# 1}

a. Meningioma

Figs. (a-c) show an abnormal signal intensity area in Right parasaggital region, showing homogenous post contrast enhancement with dural tail, this represents meningioma.

b. For treatment planning, multidisciplinary panel consultation is encouraged. Patients are stratified by presence or absence of symptoms and the tumor size. Most asymptomatic patients with small tumors $(<3 \mathrm{~cm})$ are best managed with observation, else patients should go for surgical resection whenever possible. Non surgical candidates should undergo Radiation (EBRT or SRS).

\section{ANSWER TO QUESTION \# 2}

This is not disease progression. Upon initiation of ibrutinib there is a transient increase in absolute lymphocyte count, which does not signify disease progression. This onset of isolated lymphocytosis occurs during the first few weeks of ibrutinib therapy and may persist for several weeks on treatment. 